\title{
Kidney surveillance in the spotlight: contrast-induced acute kidney injury illuminated
}

\author{
Simon J. Atkinson \\ Department of Biology, Indiana University - Purdue University Indianapolis, Indianapolis, Indiana, USA.
}

\begin{abstract}
Acute kidney injury comprises a heterogeneous group of conditions characterized by a sudden decrease in renal function over hours to days. Contrast-induced acute kidney injury (CI-AKI) is caused by radiographic contrast agents used in diagnostic imaging. In the current issue of the $J C I$, Lau et al. use a mouse model of $\mathrm{Cl}-\mathrm{AKI}$ to study the role of resident and infiltrating phagocytes, recruited leukocytes, and tubular cells in the immune surveillance response to contrast agents. This study has the potential to provide innovative therapies for human CI-AKI.
\end{abstract}

\section{Acute kidney injury}

Acute kidney injury (AKI) is an abrupt decline in kidney function that occurs over a few hours or a few days, is common in people who are already hospitalized, and is associated with significant morbidity and mortality. Multiple factors predispose to development of AKI, and treatment options, other than supportive interventions, are few (1). In addition to being associated with ischemic injury and sepsis, AKI can also result from the toxicity of therapeutic agents, such as aminoglycoside antibiotics or cisplatin chemotherapy. New work from Lau et al. in this issue of the JCI (2) examines the mechanism of contrast-induced AKI (CI-AKI), a form of AKI observed in patients receiving intravascular iodinated contrast media for diagnostic imaging studies. While the risk associated with the use of contrast media is a matter of current debate $(3,4)$, there is clear evidence for injury resulting from contrast administration in both animal models and humans, especially in patients with risk factors such as underlying chronic kidney disease. The mechanism described by Lau and colleagues is notable for the involvement of multiple cell types, including tubular epithelial cells and both resident and circulating leukocytes, all of which are required for the development of CI-AKI. This is consistent with emerging multifactorial paradigms for the development of AKI from various causes (5) and suggests both opportunities and challenges for the development of rationally designed therapeutic interventions.

\section{Animal models to study pathophysiology of Cl-AKI}

A major challenge in studies of AKI has been the applicability of animal models of AKI to what is observed in patients (6). Lau et al. use a mouse model of contrast agent administered to volume-depleted animals, chosen since volume depletion has been well documented as a contributing factor to the development of CI-AKI (7). This is clearly not a perfect model, since it (or any animal model, for that matter) cannot include all the complex and variable comorbidities, such as underlying cardiovascular disease, that are inevitable in patients referred for imaging studies. Nevertheless, there is some reassurance to be drawn from their inclusion of data from a prospective

Related Article: p. 2894

Conflict of interest: SJA is a coinventor on patent applications related to the use of hydrodynamic fluid delivery for treatment of kidney injury.

Reference information: J Clin Invest. 2018;128(7):2754-2756. https://doi.org/10.1172/JCI121741.

study of a small cohort of patients undergoing coronary angiography that are consistent with the mechanisms shown to be active in the mouse experiments.

A variety of explanations have been invoked to account for the pathophysiology of CI-AKI, with proposed mechanisms involving physiological changes such as medullary ischemia, high levels of reactive oxygen species, and toxicity of contrast media when taken up by tubular epithelial cells (8). Although related factors are also implicated in AKI from other causes, the broad AKI field has also embraced the idea that inflammatory processes induced by inappropriately exuberant activation of immune responses are major contributors, and that both resident and infiltrating leukocytes are involved (5). Lau et al. show that this paradigm extends to CI-AKI and, in so doing, show that immune activation happens in distinct compartments and depends on uptake of contrast both by tubular epithelial cells and by resident and infiltrating phagocytes.

\section{Mechanism of contrast-induced immune activation}

Their first challenge was to identify a plausible molecular mechanism by which contrast might cause immune activation. Inflammasomes are multisubunit complexes that are now recognized to be important players in both infection and the pathological inflammation that characterizes diabetes, cardiovascular disease, cancer, and a number of kidney diseases. Inflammasomes induce inflammatory responses or cell death by bringing together activator molecules capable of sensing a variety of danger signals such as pathogens that have invaded a cell, or danger-associated molecular patterns (DAMPs) released from dead or dying cells, together with effector proteases of the caspase family (9). One such inflammasome activator, Nod-like receptor pyrin containing 3 (Nlrp3), responds to a variety of stimuli, including ATP, alterations in 
A Hydrated state

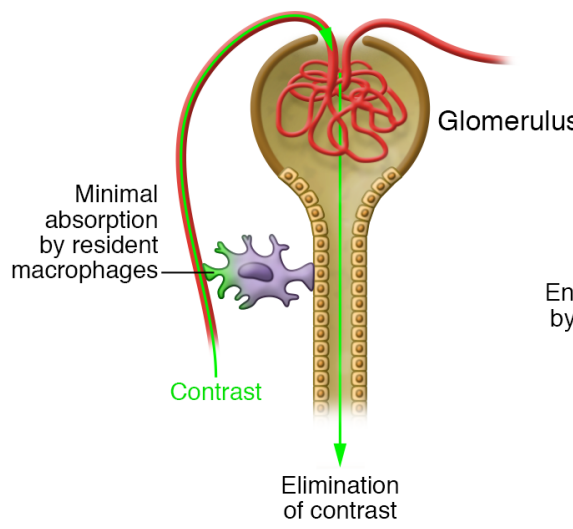

B Volume-depleted state

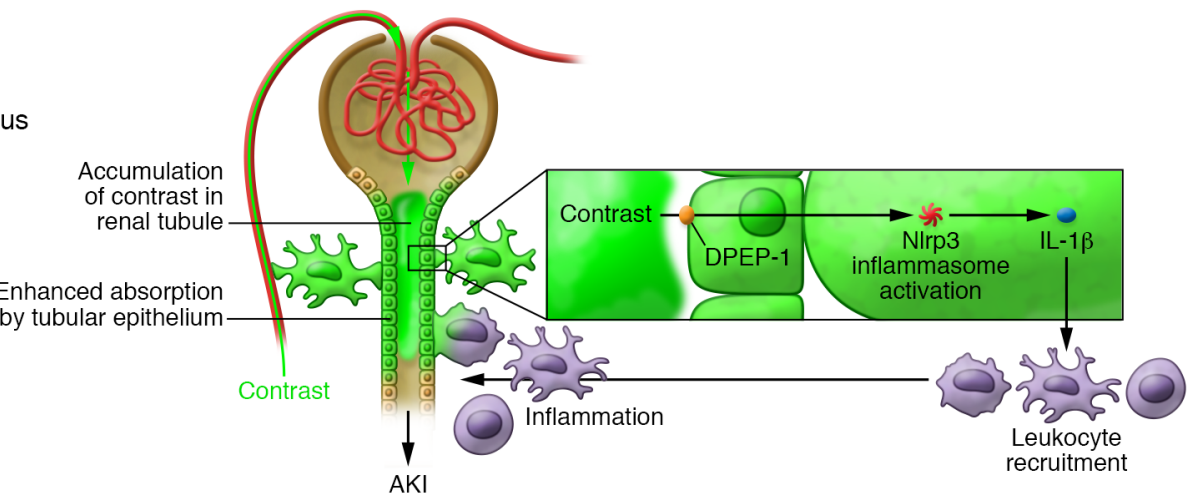

Figure 1. Dependence of contrast distribution and immune activation on volume status. In the hydrated state (A), contrast is filtered and passes into the tubule lumen but does not accumulate and is not reabsorbed. Consequently, the inflammasome in infiltrating leukocytes is not stimulated, and there is insufficient immune activation to induce a prolific leukocyte infiltration and strong inflammatory response. In contrast (B), in the volume-depleted state, filtered contrast accumulates in the tubule lumen and is reabsorbed, resulting in stimulation of infiltrating leukocytes in addition to resident macrophages, leading to a massive influx of leukocytes and an excessive inflammatory response that ultimately precipitates AKI. DPEP-1, dipeptidase-1.

intracellular potassium, and particulate matter, and has previously been shown to be an immune activator in kidney diseases (see, for example, ref. 10). Among the downstream effects induced by Nlrp3 inflammasomes is caspase-1-dependent activation of IL-1 $\beta$ via the so-called canonical pathway; noncanonical pathways activate various forms of cell death, including apoptosis. Lau et al. showed that this is also the case in their mouse model: volumedepleted $\mathrm{Nlrp3}^{-/}$mice were protected against the injury induced by contrast in their $\mathrm{Nlrp3}^{+/+}$counterparts. But in what cells does Nlrp3 inflammasome activation trigger the initiation of the injury process? Although contrast media have been shown to have direct toxic effects on tubular epithelial cells, and the Nlrp3 inflammasome is able to induce apoptosis via a noncanonical pathway, Nlrp3-dependent apoptosis alone did not account for the cytotoxic effects of contrast media seen in cell culture, and CI-AKI did not result from Nlrp3-dependent mechanisms intrinsic to the epithelial cells alone. However, contrast agent strongly activated the Nlrp3 inflammasome in leukocytes, producing IL-1 $\beta$ activation.

\section{Live imaging of}

pathophysiological processes using intravital microscopy

Intravital microscopy is a powerful technique that allows the spatial dynamics of physiological and pathophysiological processes to be followed in a live animal (11). In this study, the distribution of a fluorescently labeled contrast agent was traced by intravital microscopy, showing that the agent was present in peritubular capillaries and was filtered in the glomerulus, appearing in the lumen of the kidney tubules, regardless of volume status. Moreover, resident renal phagocytes, components of the kidney's intrinsic immune surveillance mechanism, were seen to internalize the contrast agent and demonstrated activation of the canonical pathways downstream of the Nlrp3 inflammasome. However, the accumulation of contrast in the tubule lumen and the dynamics of infiltrating leukocytes were markedly different depending on the volume status of the animals (Figure 1). In well-hydrated mice (not susceptible to CI-AKI), the contrast was rapidly cleared from the tubule lumen, whereas in volume-depleted animals (susceptible to CI-AKI), contrast tended to accumulate in patches in the tubule, with evidence of reabsorption. An Nlrp3dependent influx of leukocytes coincided with the accumulation and reabsorption of contrast, suggesting that this is an essential trigger of immune activation leading to CI-AKI. Indeed, infiltrating leukocytes were seen to crawl over the epithelial cells in the tubular regions where contrast accumulated, and these infiltrating leukocytes also showed internalization of contrast and activation of
Nlrp3 inflammasome-dependent canonical pathways. Reabsorption of contrast from the tubule and its delivery to the interstitium is necessary for its uptake by infiltrating leukocytes, and so the tubular epithelial cells, by taking up contrast that has accumulated in the tubule, retain their starring role in the CI-AKI injury mechanism. Lau et al. showed that interfering with the ability of the tubular cells to internalize contrast is a potential point of therapeutic intervention that can block immune activation and the resulting progression to CI-AKI.

\section{Lessons from the study}

There are several noteworthy outcomes of this study. First, there are three essential steps in the onset of CI-AKI: activation of the Nlrp3 inflammasome in the kidney's resident leukocytes, tubular accumulation and reabsorption of contrast, and, finally, recruitment of infiltrating leukocytes and activation of their Nlrp3-dependent canonical signaling. This suggests that there has been some selection against inappropriate activation of these inflammatory mechanisms. Another important outcome is the clear mechanistic explanation for the contribution of volume status to the likelihood of AKI in patients receiving contrast. Volume depletion is necessary for the tubular accumulation and reabsorption, since it allows activation of infiltrating leukocytes, and is therefore necessary for progression to CI-AKI. A 
caveat is that this apparently finely regulated system may well behave differently in patients with the comorbidities that are common in those who are candidates for imaging studies, particularly when these comorbid conditions involve the development of a sterile inflammatory disease state. This may compromise the effectiveness of treatment interventions such as blocking contrast uptake by tubular epithelial cells using dipeptidase-1 inhibitors, as employed by Lau et al. Finally, this study shows that the underlying mechanisms of CI-AKI revolve around dysregulated inflammatory responses in the kidney and thus are consistent with the prevailing thinking on other forms of AKI, perhaps suggesting that there are prospects for new therapeutic approaches that will be effective in AKI broadly.
Address correspondence to: Simon J. Atkinson, 755 W. Michigan Street, UL-1140, Indianapolis Indiana 46202, USA. Phone: 317.274.1020; Email: satkinso@iupui.edu.

1. Zuk A, Bonventre JV. Acute kidney injury. Annu Rev Med. 2016;67:293-307.

2. Lau A, et al. Renal immune surveillance and dipeptidase- 1 contribute to contrastinduced acute kidney injury. J Clin Invest. 2018;128(7):2894-2913.

3. Rudnick MR, Leonberg-Yoo A. The death of contrast-induced nephropathy is premature ASN Kidney News. https://www.kidneynews. org/kidney-news/features/the-death-ofcontrast-induced-nephropathy-is-premature. Accessed May 7, 2018.

4. Chen TK, Fine DM. Contrast-induced nephropathy: is the concern exaggerated? ASN Kidney News. https://www.kidneynews.org/kidneynews/features/contrast-induced-nephropathy-isthe-concern-exaggerated. Accessed May 7, 2018.
5. Rabb H, et al. Inflammation in AKI: current understanding, key questions, and knowledge gaps. J Am Soc Nephrol. 2016;27(2):371-379.

6. Agarwal A, et al. Cellular and molecular mechanisms of AKI. JAm Soc Nephrol. 2016;27(5):1288-1299.

7. Solomon R, Werner C, Mann D, D'Elia J, Silva P. Effects of saline, mannitol, and furosemide on acute decreases in renal function induced by radiocontrast agents. $N$ Engl JMed. 1994;331(21):1416-1420.

8. Azzalini L, Spagnoli V, Ly HQ. Contrastinduced nephropathy: from pathophysiology to preventive strategies. Can J Cardiol. 2016;32(2):247-255.

9. Rathinam VA, Fitzgerald KA. Inflammasome complexes: emerging mechanisms and effector functions. Cell. 2016;165(4):792-800.

10. Vilaysane A, et al. The NLRP3 inflammasome promotes renal inflammation and contributes to CKD. J Am Soc Nephrol. 2010;21(10):1732-1744.

11. Dunn KW, et al. Functional studies of the kidney of living animals using multicolor twophoton microscopy. Am J Physiol, Cell Physiol. 2002;283(3):C905-C916. 